\title{
Variability of the Expression Patterns of Neuroinflammatory Genes in Mononuclear Cells of Peripheral Blood in Amyotrophic Lateral Sclerosis
}

\author{
M. V. Ivanova ${ }^{a, 1}$, A. S. Voronkova ${ }^{a}$, V. S. Sukhorukov ${ }^{a}$, and M. N. Zakharova ${ }^{a}$ \\ ${ }^{a}$ Research Center of Neurology, Moscow, Russia \\ Received December 31, 2020; revised January 5, 2021; accepted January 6, 2021
}

\begin{abstract}
Amyotrophic lateral sclerosis (ALS) is a neurodegenerative disease with steadily progressing death of motor neurons in the brain and spinal cord. The disease is incurable and results in the patient's death within 3-5 years on average. The mechanisms of initiation, progression, and spread of the pathological process remain unclear. It seems that inflammatory reactions may be important for disease progression but this is not certain. We performed multiplex analysis of the expression of neuroinflammatory genes in mononuclear cells of peripheral blood from patients with different rates of ALS progression $(n=22)$ and compared these data with those observed in healthy volunteers. We found that the expression of 14 genes, specifically $B A X, C L N 3$, PLEKHM1, AKT1, LAMP1, RAC2, VAV1, MPG, TFG, BRD2, CSK, MSN, GBA, and VIM, differed between the groups of patients and healthy volunteers $(p<0.05 ; q<0.05)$. Genes associated with autophagia, apoptosis, adaptive immunity, and growth factor cascades prevail among these genes. We did not find any substantial differences in gene expression between patients with rapid and slow ALS progression. We revealed a subgroup of patients who exhibited significantly different expression of 208 or 262 genes compared to other ALS patients or healthy volunteers, respectively $(p<0.05 ; q<0.05)$. Our data show the importance of not only central but also peripheral inflammatory reactions in the development of the pathological process associated with ALS.
\end{abstract}

Keywords: amyotrophic lateral sclerosis, neuroinflammation, neurodegeneration, apoptosis, autophagia, adaptive immunity, progression rates, multiplex analysis of expression

DOI: $10.1134 / \mathrm{S} 1819712421020070$

\section{INTRODUCTION}

Amyotrophic lateral sclerosis (ALS) is a neurodegenerative disease with steadily progressing death of motor neurons, which results in the development of spasticoatrophic damage to skeletal muscles. The median age of disease onset is 55 years although the disease incidence is growing among young people [1] which is probably related to deterioration of the environmental situation. As a rule, the disease starts at one anatomical level and after that the pathological process gradually spreads and finally involves vital respiratory muscles resulting in the patient's death within 3-5 years on average [2]. In ALS, the rates of progression of the pathological process are very different. For a long time, Russian and foreign scientists have been searching for diagnostic and prognostic markers of the disease [3], but this issue remains unresolved, which makes it difficult for the patient to make decisions on life planning. Efficient methods for ALS treatment have still not been developed.

\footnotetext{
${ }^{1}$ Corresponding author; address: Volokolamskoe sh., 80, Moscow, 125367 Russia; phone: 8(495)490-24-10; e-mail: fspsbl@gmail.com.
}

The mechanisms of initiation, progression, and spread of the pathological process remain unclear. According to modern ideas, genetic factors play an important role. Five to $15 \%$ of ALS cases are familial whereas others are sporadic; however, even in the latter case, potentially pathogenic mutations can often be observed [4]. Impaired protein homeostasis with accumulation of pathological protein aggregates and RNA processing play a leading role among the pathophysiological processes which are important for ALS development. Some impairments of cytoskeleton dynamics, structure and function of axons, endosomal transport, and DNA repair, as well as autophagia, excitotoxicity, oxidative stress, mitochondrial dysfunction, microglia activation, and finally neuroinflammation also play a pathogenic role [5].

It seems that neuroinflammation is important for the progression of the pathological process in ALS. Protective anti-inflammatory reactions that predominantly develop at the initial stages of ALS are replaced by toxic proinflammatory reactions as the disease progresses [6]. However, the mechanisms of neuroin- 
flammation that are of key importance in ALS are currently poorly studied.

Taking this into account, examination of the expression of neuroinflammation-associated genes in ALS can help to reveal new diagnostic and prognostic biomarkers and targets for therapeutic treatment. In the present study, we aimed to perform multiplex analysis of the expression of genes associated with a wide spectrum of neuroinflammatory processes in patients with different rates of ALS progression and healthy volunteers.

\section{MATERIALS AND METHODS}

Patients. The inclusion criteria were diagnosis of ALS according to the revised El Escorial criteria [7], age older than 20 years, rapid or slow rate of disease progression (see below), bulbar, cervical-thoracic or lumbo-sacral forms of ALS, and the presence of signed informed consent to participate in the study. The exclusion criteria were the presence of chronic inflammatory diseases, oncological pathology, other neurological disorders, acute infection diseases in the month prior to inclusion in the study, and intake of drugs influencing the immune system, such as corticosteroids, cytostatics, and others. Based on the exclusion criteria, the group did not include 7 patients who were potentially eligible for inclusion. One patient was excluded from analysis because they did not pass the quality control during the study on the expression of neuroinflammatory genes. Thus, 22 ALS patients and 12 healthy volunteers were included in the study. Patients were divided into two subgroups depending on the rate of disease progression during the 12 months prior to inclusion in the study. Group 1 consisted of patients with slow progression, i.e. a decrease in the score on the functional assessment scale for ALSFRS-R of no more than 2 points. Group 2 consisted of patients with rapid progression, i.e. a decrease in the score on the scale for ALS-FRS-R of 10 points and more. Patients did not suffer from chronic autoimmune, infection or oncological diseases or acute infections for at least one month prior to inclusion in the study.

RNA extraction. RNA was extracted from peripheral blood mononuclear cells (PBMC). Blood samples were collected using EDTA-containing BD Vacutainer tubes (BD, United States) and treated within $1 \mathrm{~h}$ of sampling. PBMC were separated in the density gradient using Histopaque-1077 ficoll (SigmaAldrich, United States). The blood samples were carefully applied to the ficoll layer in a $15 \mathrm{~mL}$ tube. The tubes were centrifuged at $400 \mathrm{~g}$ for $30 \mathrm{~min}$ at room temperature. After centrifugation, the PBMC layer was transferred into a sterile tube, washed with $10 \mathrm{~mL}$ phosphate buffer containing 1\% FBS and centrifuged again at $300 \mathrm{~g}$ for $10 \mathrm{~min}$. The supernatant was discarded, the cells were resuspended in $1 \mathrm{~mL}$ of the RPMI medium and counted. Aliquots, containing 3-
$5 \times 10^{6} \mathrm{PBMC}$, were frozen at $-80^{\circ} \mathrm{C}$ and stored until RNA extraction. RNA was extracted from one aliquot using a Total RNA Purification Plus Kit (Norgern Biotek, Canada) according to the manufacturer's protocol. RNA concentration was measured using a NanoDrop2000C spectrophotometer (ThermoScientific, United States) and corrected to the $60 \mathrm{ng} / \mu \mathrm{L}$ level using a vacuum concentrator. For all the samples the $\mathrm{OD}_{260 / 280}$ ratio was $1.72-2.10$. RNA samples were stored for no longer than 1 month at $-80^{\circ} \mathrm{C}$.

Multiplex analysis of neuroinflammatory gene expression. Neuroinflammatory gene expression was estimated using the NanoString technique (NanoString Technologies, WA, United States). This technology is based on unique barcoding of target RNA using fluorescent probes. In the current study we used a standard panel of neuroinflammatory genes, the nCounter Human Neuroinflammation panel, which includes 770 genes including 758 signature genes and 12 constitutive genes. The samples were analyzed using a nCounter Analysis System device (NanoString Technologies, United States) according to the manufacturer's instructions. Each sample contained at least $300 \mathrm{ng}$ of RNA. Initial data analysis was performed using the nSolver package (version 4.0) software. The primary data were normalized using 6 positive controls and 12 constitutive genes included in the panel. After that an Advanced Analysis module was used for analysis of the expression. The data from the nCounter system are expressed as arbitrary units, which reflect the concentration of target RNA molecules in the sample. The investigator was blinded to the conditions, i.e., did not know which samples belonged to particular groups of patients.

Statistical analysis. Statistical analysis of data was performed using the IBM SPSS Statistics 23 and GraphPad Prizm 8 software. Clinical characteristics and relation to a particular group were encoded, and the experimenter did not have access to patient data during the statistical analysis. Log2-normalized data were used for analysis. The correspondence of data to normal distribution was revised using the Kolmogorov-Smirnov test. The distribution of data in the groups of healthy volunteers $(0)$, patients with slow or rapid ALS progression as well as in the groups with patterns 1 or 2 of disease manifestation was normal; therefore, for differential gene expression analysis we applied one-way analysis of variances in those groups with correction for sex and age. The differential gene expression was considered as significant at $p<0.05$. In order to exclude false-positive results the BnjaminiHochberg correction was applied to multiple comparisons with a threshold value of $q<0.05$. Bonferroni correction was applied for paired group comparisons in the post hoc analysis of the differential gene expression data. The differences were considered as significant at $p<0.05$. Spearman rank correlation coefficients were calculated in order to reveal correlations 
Table 1. Clinical characteristics of the groups

\begin{tabular}{l|c|c|c|c}
\hline \multicolumn{1}{c|}{ Index } & $\begin{array}{c}\text { All ALS patients } \\
(n=22)\end{array}$ & $\begin{array}{c}\text { Rapidly progressing } \\
(n=11)\end{array}$ & $\begin{array}{c}\text { Slowly progressing } \\
(n=11)\end{array}$ & $\begin{array}{c}\text { Healthy volunteers } \\
(n=12)\end{array}$ \\
\hline Age, Me [Q1; Q3] & $52.5[45,2 ; 64.5]$ & $57[49 ; 69]$ & $51[36.5 ; 56]$ & $49.5[41.25 ; 56]$ \\
Sex M/F, \% & $50 / 50$ & $63.6 / 36.4$ & $36.4 / 63.6$ & $42 / 52$ \\
Disease duration, Me [Q1; Q3] & $18.5[14.2 ; 37.5]$ & $15[9 ; 18]$ & $36[19 ; 48.5]$ & - \\
Stage of disease (2/3/4) & $8 / 7 / 7$ & $0 / 4 / 7$ & $8 / 3 / 0$ & - \\
Form (B/CT/LS) & $5 / 12 / 5$ & $4 / 6 / 1$ & $1 / 6 / 4$ & - \\
ALSFRS-R & $38[35 ; 43.5]$ & $35[24.5 ; 37]$ & $44[41 ; 44.5]$ & - \\
VLC (\%PVLC), Me [Q1;Q3] & $89.8[69.4 ; 100.8]$ & $70.2[60 ; 99.7]$ & $95[81.6 ; 101]$ & - \\
BMI, Me [Q1;Q3] & $27.2[23.5 ; 35.2]$ & $25.7[21.3 ; 35.7]$ & $29.8[26.3 ; 31.4]$ & - \\
\hline
\end{tabular}

Me, median; Q1, quartile 1; Q3, quartile 3; n, number of patients; M, male; F, female; B, bulbar form; CT, cervical-thoracic form; LS, lumbar-sacral form; VLC, vital lung capacity; PVLC, proper vital lung capacity; BMI, body mass index.

between gene expression and clinical features of the disease. Correlations were considered as significant at $p<0.05$.

\section{RESULTS}

Clinical characteristics of patients. Twelve healthy volunteers and 22 ALS patients were included in the study, of which 11 patients exhibited a rapid progression rate during the previous year with a 10-point decrease according to the ALS-FRS-R scale and the other 11 patients exhibited a slow progression rate with a 1-2-point decrease according to the ALS-FRS-R scale. The clinical characteristics of the groups studied are shown in Table 1. We did not find any statistical differences in sex, age, frequency of occurrence of various forms of the disease, vital lung capacity (VLC), and body mass index (BMI) between the groups. We found a shorter duration of the disease $(p=0.002)$, a lower value according to the ALS-FRS-R scale $(p<$ $0.001)$, and a higher stage of the disease $(p<0.001)$ in the group of patients with a rapid progression rate, indicating a worse functional condition of the patients. The group of patients with a rapid progression rate consisted of more patients with the bulbar form and less patients with the lumbo-sacral form compared to the group with slow progression. This was not surprising because the onset of bulbar disorders in ALS is considered as prognostically unfavorable [7]. However, the anatomical level involved in the onset of the disease did not affect the homogeneity of the groups due to the common pathophysiological process and thus, did not affect the results of statistical analysis.

Expression of neuroinflammatory genes in ALS patients. Heat mapping was used for visualization of the differences in the expression of neuroinflammatory genes. According to this, there was a large pool of genes which were differentially expressed in healthy volunteers and ALS patients. There were also groups of genes with differential expression in the patient groups with various rates of ALS progression (Fig. 1).
However, statistical analysis revealed the differences in the expression levels of only 14 genes in the groups of patients with rapid and slow progression of the disease and healthy volunteers. They included the $B A X$, CLN3, PLEKHM1, AKT1, LAMP1, RAC2, VAV1, $M P G, T F G, B R D 2, C S K, M S N, G B A$, and VIM genes (Table 2). The most widely represented functional groups with a statistically significant difference in expression were genes associated with autophagy (CLN3, AKT1, LAMP1, RAC2, VAV1, CSK, MSN), apoptosis (BAX, $A K T 1, T F G, G B A)$, and adaptive immunity and growth factors $(A K T 1, R A C 2, V A V 1$, $C S K$ ). Paired comparison of groups showed that expression of all the mentioned genes except $B R D 2$ differed between the ALS patients and healthy volunteers but not between the patients with rapid and slow rates of ALS progression. Expression of the BRD2 gene was increased in the group of patients with rapid compared to the group with slow ALS progression or healthy volunteers. The $B R D 2$ gene belongs to a functional group related to epigenetic regulation. Previously, BRD2 was not associated with ALS. It was associated with the development of the nervous system during embryogenesis, juvenile myoclonic epilepsy, obesity, and certain hemoblastoses [8-10].

Correlation between the expression of neuroinflammatory genes and clinical characteristics. Correlation analysis was applied to reveal the relationships between the expression of neuroinflammatory genes and clinical characteristics of the disease in the 22 ALS patients involved in the study. We found statistically significant moderate positive $(r=0.5-0.75)$ correlations between the expression of genes encoding $B R D 2$ $(r=0.535, p=0.01)$, related to epigenetic regulation, and $F Y N(r=0.575, p=0.005)$, related to the cascades of innate and adaptive immunity, functioning of microglia, growth factors, angiogenesis, and autophagia, with the disease stage. Moderate negative correlations were observed between the ALS-FRS-R scale values and the expression of genes encoding $K D M 3 A$ $(r=-0.623, p=0.002)$ associated with epigenetic reg- 


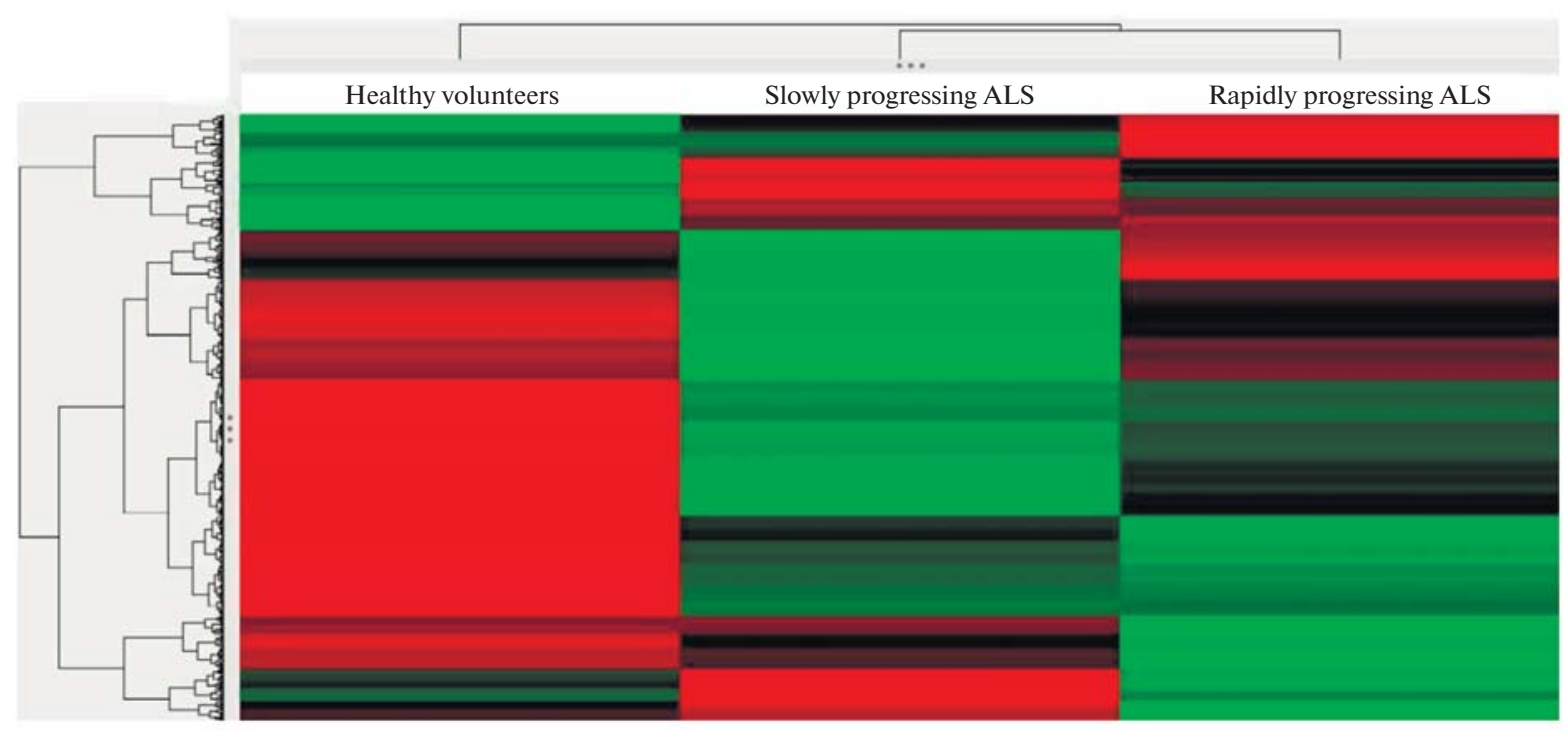

Fig. 1. Expression of neuroinflammatory genes in ALS patients and healthy volunteers. Bars represent mean values of gene expression in the groups: group 0, healthy volunteers; 1, slow progressing ALS in the last 12 months; 2, rapidly progressing ALS in the last 12 months. Rows show expression of specific genes: black, mean value in total sample of patients; red, increased expression level compared to the mean; green, decreased expression level.

ulation, PILRB $(r=-0.512, p=0.015)$ involved in the innate immunity response, and TSPAN18 $(r=$ $-0.529, p=0.011$ ) associated with microglia functioning. A decrease in the ALS-FRS-R scale value within the last 12 months positively correlated with $B R D 2$ $(r=0.588, p=0.004), C T S W(r=0.526, p=0.012)$ secreted by cytotoxic cells, and $K D M 3 A(r=0.542, p=$ $0.009)$. Most of the statistically significant correlations were observed between the expression of neuroinflammatory genes and the disease duration. Expression of the genes encoding $C 6(r=0.547, p=0.008)$, $C C N G 2(r=0.514, p=0.014), C D 47(r=0.543, p=$ $0.009), C L D N 5(r=0.501, p=0.017), C L I C 4(r=$ $0.628, p=0.002), C O A 5(r=0.501, p=0.018)$, COL6A3 $(r=0.527, p=0.012), D N A 2(r=0.510, p=$ $0.015), \operatorname{ESAM}(r=0.525, p=0.012), I K B K B(r=$ $0.516, p=0.014)$, PROS1 $(r=0.651, p=0.001)$, RAB6B $(r=0.629, p=0.002)$, and TMEM64 $(r=$ $0.675, p=0.001)$ positively correlated with the disease duration. Among them, the most widely represented genes were those associated with microglial activation and matrix remodeling. The expression of $D D B 2$ associated with DNA damage negatively correlated with the duration of the disease.

Inflammatory pattern of expression in a group of patients. Analysis of heat maps and volcano plots of neuroinflammatory gene expression in ALS patients revealed two patterns of expression: pattern 1 (12 patients) and pattern 2 (7 patients) (Fig. 2). Statistical analysis of neuroinflammatory genes expression in patients with pattern 1 , pattern 2 , and healthy volunteers showed 292 differentially expressed genes. Paired post hoc comparison of groups showed pattern 2 had the most significant difference compared to the other groups. The patients with pattern 2 had statistically significant differences in the expression of 208 and 262 genes compared to the patients with pattern 1 or healthy volunteers, respectively. On the contrary, the patients with pattern 1 significantly differed from the group of healthy volunteers in expression of only 49 genes. The main functional groups of genes that were differentially expressed in the patients with pattern 2 compared to the other patterns of expression were the genes associated with innate or adoptive immunity, microglia functioning, apoptosis, autophagia, and growth factors. However, we did not observe any substantial differences in the clinical characteristics of patients with patterns of gene expression 1 and 2 . The rates of disease progression were not related to the expression pattern either.

\section{DISCUSSION}

Here, we found neuroinflammatory genes, the expression of which was different in peripheral blood mononuclear cells in ALS patients and healthy individuals. Specifically, these genes were associated with autophagia, apoptosis, adaptive immunity response or growth factor signaling cascades. Two different patterns of neuroinflammatory gene expression were revealed in ALS patients. One of them was similar to the expression pattern observed in healthy individuals whereas the other had differences in the expression of genes related to innate and adaptive immunity, microglia function, autophagia and apoptosis, and growth factor cascades.

More and more studies point to the role of systemic inflammation and immune response in ALS patients 
Table 2. Differentially expressed genes in ALS patients and healthy volunteers

\begin{tabular}{|c|c|c|c|c|c|c|c|}
\hline Gene & Group & Mean & $\begin{array}{c}\text { Standard } \\
\text { error of mean }\end{array}$ & Fold change & F(ANOVA) & $p$ & $q$ \\
\hline \multirow{3}{*}{$B A X$} & 0 & 1065.54 & 38.03 & 1 & \multirow{3}{*}{20.21} & \multirow{3}{*}{0.0000024} & \multirow{3}{*}{0.001596} \\
\hline & 1 & 1357.79 & 42.59 & 1.27 & & & \\
\hline & 2 & 1449.79 & 58.98 & 1.36 & & & \\
\hline \multirow{3}{*}{ CLN3 } & 0 & 87.02 & 6.57 & 1 & \multirow{3}{*}{17.97} & \multirow{3}{*}{0.0000066} & \multirow{3}{*}{0.0021945} \\
\hline & 1 & 142.04 & 10.18 & 1.63 & & & \\
\hline & 2 & 141.30 & 8.66 & 1.62 & & & \\
\hline \multirow{3}{*}{ PLEKHM1 } & 0 & 265.07 & 15.49 & 1 & \multirow{3}{*}{16.63} & \multirow{3}{*}{0.0000124} & \multirow{3}{*}{0.0027487} \\
\hline & 1 & 364.52 & 19.43 & 1.38 & & & \\
\hline & 2 & 393.70 & 18.99 & 1.49 & & & \\
\hline \multirow{3}{*}{$A K T 1$} & 0 & 363.47 & 18.59 & 1 & \multirow{3}{*}{12.97} & \multirow{3}{*}{0.0000808} & \multirow{3}{*}{0.0107255} \\
\hline & 1 & 508.90 & 33.61 & 1.40 & & & \\
\hline & 2 & 529.28 & 31.61 & 1.46 & & & \\
\hline \multirow{3}{*}{$L A M P 1$} & 0 & 1274.60 & 57.47 & 1 & \multirow{3}{*}{12.74} & \multirow{3}{*}{0.0000917} & \multirow{3}{*}{0.0107255} \\
\hline & 1 & 1665.57 & 87.34 & 1.31 & & & \\
\hline & 2 & 1720.85 & 76.17 & 1.35 & & & \\
\hline \multirow{3}{*}{ RAC2 } & 0 & 1393.33 & 85.17 & 1 & \multirow{3}{*}{12.55} & \multirow{3}{*}{0.0001017} & \multirow{3}{*}{0.0107255} \\
\hline & 1 & 1896.25 & 122.81 & 1.36 & & & \\
\hline & 2 & 1975.77 & 82.79 & 1.42 & & & \\
\hline & 0 & 899.20 & 32.32 & 1 & & & \\
\hline$V A V 1$ & 1 & 1085.42 & 29.45 & 1.21 & 12.36 & 0.0001129 & 0.0107255 \\
\hline & 2 & 1072.64 & 29.86 & 1.19 & & & \\
\hline & 0 & 172.94 & 8.72 & 1 & & & \\
\hline$M P G$ & 1 & 217.20 & 10.56 & 1.26 & 12.02 & 0.0001364 & 0.0113383 \\
\hline & 2 & 235.33 & 9.04 & 1.36 & & & \\
\hline & 0 & 339.05 & 9.70 & 1 & & & \\
\hline$T F G$ & 1 & 410.41 & 17.44 & 1.21 & 11.02 & 0.0002423 & 0.0165718 \\
\hline & 2 & 425.99 & 16.53 & 1.26 & & & \\
\hline & 0 & 2109.94 & 94.99 & 1 & & & \\
\hline BRD2 & 1 & 2307.51 & 65.45 & 1.09 & 10.97 & 0.0002492 & 0.0165718 \\
\hline & 2 & 2665.99 & 89.24 & 1.26 & & & \\
\hline & 0 & 2296.49 & 93.43 & 1 & & & \\
\hline$C S K$ & 1 & 2799.89 & 78.46 & 1.22 & 10.32 & 0.0003678 & 0.0222352 \\
\hline & 2 & 2763.49 & 97.28 & 1.20 & & & \\
\hline & 0 & 5692.86 & 234.16 & 1 & & & \\
\hline$M S N$ & 1 & 6905.87 & 246.57 & 1.21 & 9.85 & 0.0004879 & 0.0269683 \\
\hline & 2 & 6950.10 & 220.10 & 1.22 & & & \\
\hline & 0 & 514.01 & 35.32 & 1 & & & \\
\hline$G B A$ & 1 & 696.76 & 25.83 & 1.36 & 9.72 & 0.0005272 & 0.0269683 \\
\hline & 2 & 678.12 & 27.54 & 1.32 & & & \\
\hline & 0 & 10900.21 & 529.63 & 1 & & & \\
\hline$V I M$ & 1 & 13737.53 & 694.63 & 1.26 & 8.74 & 0.0009768 & 0.046398 \\
\hline & 2 & 15899.32 & 1440.21 & 1.46 & & & \\
\hline
\end{tabular}

Group 0, healthy volunteers; 1, slowly progressing ALS; 2, rapidly progressing ALS. Data are presented as arbitrary units reflecting the content of target RNA molecules in the sample. 
(a)

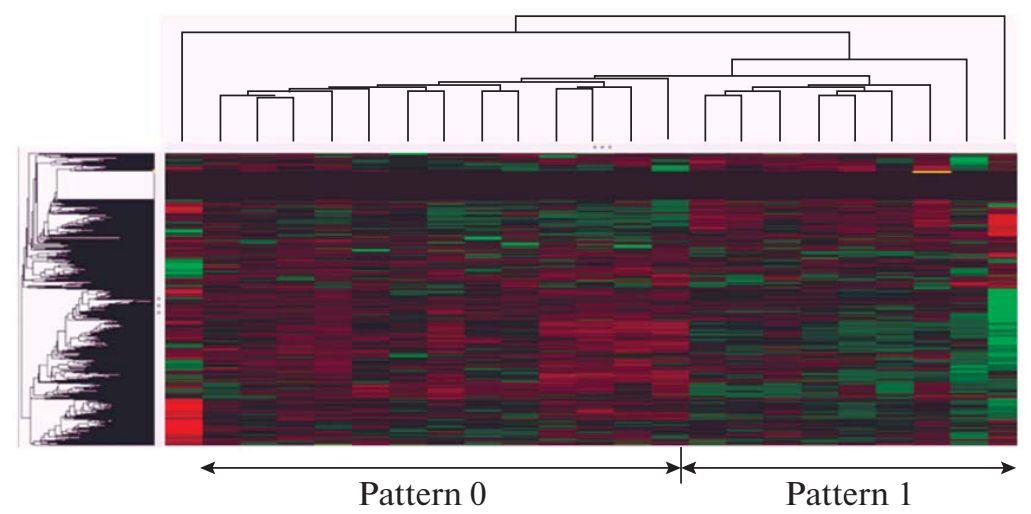

(b)

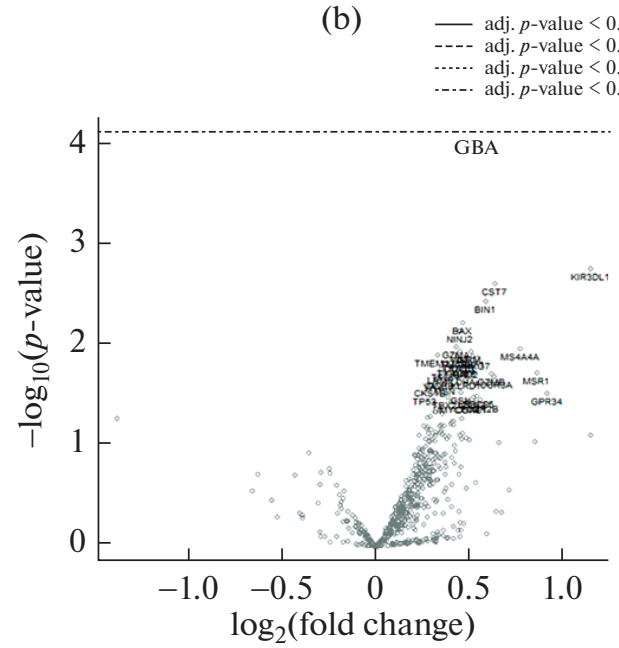

(c)
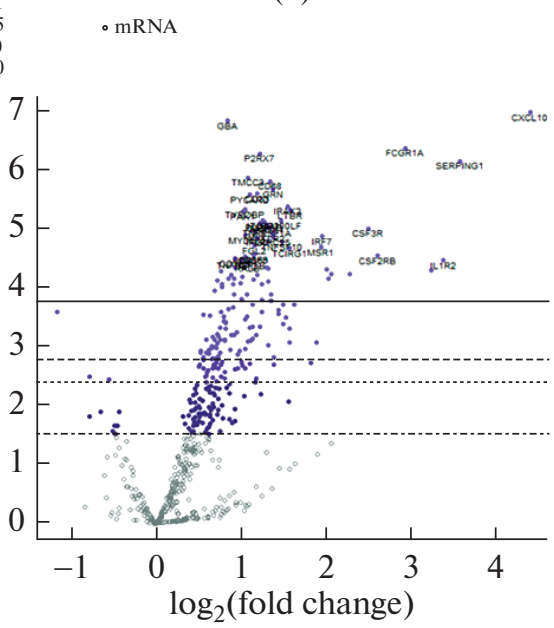

Fig. 2. Different profiles of neuroinflammatory gene expression in ALS patients. (a) Heat map of neuroinflammatory genes expression in ALS patients. Gene expression value for individual patients are presented in columns. Specific levels of gene expression are presented in rows according to the following color scheme: black, mean value in total patient sample; red, enhanced level of expression compared to the mean value; green, decreased level of expression. Column 1 contains data for the patient who was excluded from the study because his sample did not pass the quality control. (b) Volcano plot represents changes in the expression of genes associated with neuroinflammation in the group with profile 1 compared to healthy volunteers. (c) Volcano plot represents changes in the expression of genes associated with neuroinflammation in the group with profile 2 compared to healthy volunteers. Abscissa axis, expression, fold of changes; ordinate axis, negative decimal logarithm of the $p$-value. In (b and c), abscissa axis, expression, fold of changes; ordinate axis, negative decimal logarithm of the $p$-value. Genes that differ significantly between the groups are highlighted in blue.

[11-13]. It has been shown that in ALS, microglia and astrocytes are activated, which is neuroprotective in the early stages of the disease and proinflammatory in the later stages. In the areas of motor neuron death in the CNS, the activation of microglia and perivascular infiltration of monocytes and $\mathrm{T}$ cells are described as the main pathological changes [14]. In vivo positron emission tomography of the brain of ALS patients demonstrates signs of widespread activation of microglia [15]. Being activated due to other pathological ALS-associated reactions, astrocytes and microglia contribute to the acceleration of the rate of neuronal death. Proinflammatory cytokines secreted by these cells contribute to the processes of glutamate excitotoxicity, which links neuroinflammation and the neuronal death associated with excitotoxicity [16].

In ALS, mutations in multiple genes expressed in neurons and microglia have been reported, which lead to impairment of autophagia, activation of inflammatory pathways in microglia and astroglia, and death of motor neurons $[1,17]$. However, even in patients with sporadic ALS without known genetic mutations an increased number of proinflammatory macrophages and dysfunctional T-regulatory cells is observed [13, 18]. Several studies demonstrated decreased expression of the main marker of T-regulatory cells FOXP3 transcription factor in ALS patients as well as their capability to inhibit proliferation of other T-lympho- 
cytes, leading to activation of proinflammatory reactions. These alterations are mostly expressed in patients with a high rate of disease progression. Interestingly, during the cultivation of T-regulatory cells derived from ALS patients, their functional capacities recovered with time $[18,19]$.

When analyzing the transcriptome in twins, among whom only one had ALS, a difference in the expression of 750 genes belonging to 74 signaling pathways was revealed. Among them, there was an increase in the expression of genes belonging to 25 pathways associated with immune functions and signal transduction in cells [20]. Changes in the expression of genes associated with neuroinflammation were also found in fibroblasts from ALS patients, compared with fibroblasts of healthy people; the most pronounced differences were in the expression of genes belonging to the signaling pathways of Toll- and NOD-like receptors [12]. When analyzing the expression of neuroinflammatory genes in circulating monocytes of ALS patients, a specific proinflammatory pattern was revealed that included $I L 1 B, I L 8, F O S B, C X C L 1$, and $C X C L 2$. Zhao et al. detected more genes with different expression in the monocytes of patients with rapidly progressing ALS compared to healthy volunteers or the monocytes of patients with slowly progressing ALS. This proinflammatory state of the innate immune system indicates a possible role of monocytes/macrophages in ALS pathogenesis [13]. In the present study, we did not find any substantial differences between the patterns of neuroinflammatory gene expression in patients with rapid and slow ALS progression. This discrepancy in results may be due to the use of a different set of studied genes and biological material, as well as other criteria for assessing the rate of ALS progression. Furthermore, we did not reveal a faster rate of disease progression in patients with a specific pattern of expression of 2 neuroinflammatory genes. The clinical characteristics associated with the proinflammatory gene expression profile in PBMC in some ALS patients in our study have yet to be clarified.

How proinflammatory myeloid cells may be associated with ALS progression is still unclear. It is possible that they migrate to the CNS, since in ALS and in ALS models, an impairment of the integrity of the blood-brain barrier is shown [21]. Because they provide proinflammatory effects in the CNS, they can promote neurodegeneration. Even if they do not penetrate the CNS, they could modulate the course of the disease by increasing the severity of peripheral inflammation; if they penetrate the spleen and lymph nodes or contact peripheral blood cells then they can modulate the proinflammatory status of T cells [22], dendritic cells, and natural killers, having an indirect effect on inflammatory processes in the CNS.

\section{CONCLUSIONS}

Thus, our data show the activation of the inflammatory process in peripheral immune cells compared to healthy people. Much data point to the fact that inflammation reactions are one of the mechanisms that promotes the progression of neurodegeneration in ALS and other neurodegenerative diseases. It seems that ALS is a multistage process including a lot of pathogenetic cascades. Each step in this process contributes its own pathophysiological alterations and thus becomes an integral element in the development and progression of the disease. In our study, a group of patients was revealed with a specific pattern of changes in the expression of genes associated with neuroinflammation. Whether the expression of genes associated with neuroinflammation can be considered as a potential predictive biomarker is still unclear. Further study of inflammatory responses in ALS may become the basis for the development of new therapeutic approaches that will reduce the rate of progression of the disease.

\section{FUNDING}

This study was supported by the Russian Foundation for Basic Research, project no. 18-015-00480 A.

\section{COMPLIANCE WITH ETHICAL STANDARDS}

Conflict of interests. The authors declare no conflict of interests.

Ethical approvement. All procedures involving humans were performed in accordance with ethical standards of the institutional and national Committees on research ethics and the Helsinki Declaration of 1964 including its subsequent amendments.

Informed consent. Each participant involved in the study signed informed consent to participate in a research study.

\section{OPEN ACCESS}

This article is licensed under a Creative Commons Attribution 4.0 International License, which permits use, sharing, adaptation, distribution and reproduction in any medium or format, as long as you give appropriate credit to the original author(s) and the source, provide a link to the Creative Commons license, and indicate if changes were made. The images or other third party material in this article are included in the article's Creative Commons license, unless indicated otherwise in a credit line to the material. If material is not included in the article's Creative Commons license and your intended use is not permitted by statutory regulation or exceeds the permitted use, you will need to obtain permission directly from the copyright holder. To view a copy of this license, visit http://creativecommons.org/licenses/by/4.0/. 


\section{REFERENCES}

1. Brown, R.H. and Al-Chalabi, A., N. Engl. J. Med., 2017, vol. 377, pp. 162-172.

2. Vasil'ev, A.V., Eliseeva, D.D., Ivanova, M.V., Kochergin, I.A., Zakroishchikova, I.V., Brylev, L.V., Shtabnitskii, V.A., and Zakharova, M.N., Ann. Klinicheskoi Eksperimental'noi Nevrologii, 2018, vol. 12, pp. 76-85.

3. Guo, J., Yang, X., Gao, L., and Zang, D., Brain Behav., 2017, vol. 19, pp. e00637.

4. van Es, M.A., Hardiman, O., Chio, A., Al-Chalabi, A., Pasterkamp, R.J., and Veldink, J.H., Lancet, 2017, vol. 390, pp. 2084-2098.

5. Hardiman, O., Al-Chalabi, A., Chio, A., Corr, E.M., Logroscino, G., and Robberecht, W., Nat. Rev. Dis. Primers, 2017, vol. 3, pp. 17071.

6. Beers, D.R. and Appel, S.H., Lancet Neurol., 2019, vol. 18, pp. 211-220.

7. Brooks, B.R., Miller, R.G., Swash, M., and Munsat, T.L., Amyotroph. Lateral Scler., 2000, vol. 1, pp. 293-299.

8. Pal, D.K., Evgrafov, O.V., Tabares, P., Zhang, F., Durner, M., and Greenberg, D.A., Am. J. Hum. Genet., 2003, vol. 73, pp. 261-270.

9. Wang, F., Liu, H., Blanton, W.P., Belkina, A., Lebrasseur, N.K., and Denis, G.V., Biochem. J., 2010, vol. 425, pp. 71-83.

10. Greenwald, R.J., Tumang, J.R., Sinha, A., Currier, N., Cardiff, R.D., and Rothstein, T.L., Blood, 2004, vol. 103, pp. 1475-1484.
11. Lu, C.H., Allen, K., Oei, F., Leoni, E., Kuhle, J., and Tree, T., Neurol. Neuroimmunol. Neuroinflammation, 2016, vol. 3, pp. e244.

12. Won, Y.H., Lee, M.Y., Choi, Y.C., Ha, Y., Kim, H., and Kim, D.Y., PLoS One, 2016, vol. 11, pp. e0165290.

13. Zhao, W., Beers, D.R., Hooten, K.G., Sieglaff, D.H., Zhang, A., and Kalyana-Sundaram, S., JAMA Neurol., 2017, vol. 74, pp. 677-685.

14. Zhao, W., Beers, D.R., and Appel, S.H., J. Neuroimmune Pharmacol., 2013, vol. 8, pp. 888-899.

15. Corcia, P., Tauber, C., Vercoullie, J., Arlicot, N., Prunier, C., and Praline, J., PLoS One, 2012, vol. 7, pp. 12.

16. Lee, J., Hyeon, S.J., Im, H., Ryu, H., Kim, Y., and Ryu, H., Exp. Neurobiol., 2016, vol. 8, pp. 233-240.

17. Renton, A.E., Chiò, A., and Traynor, B.J., Nat. Neurosci., 2014, vol. 17, pp. 17-23.

18. Beers, D.R., Zhao, W., Wang, J., Zhang, X., Wen, S., and Neal, D., JCI Insight, 2017, vol. 2, pp. 5.

19. Henkel, J.S., Beers, D.R., Wen, S., Rivera, A.L., Toennis, K.M., and Appel, J.E., EMBO Mol. Med., 2013, vol. 5, pp. 64-79.

20. Tarr, I.S., McCann, E.P., Benyamin, B., Peters, T.J., Twine, N.A., and Zhang, K.Y., Sci. Rep., 2019, vol. 9, p. 1.

21. Garbuzova-Davis, S. and Sanberg, P.R., Front. Cell. Neurosci., 2014, vol. 8, p. 21.

22. Stockinger, B., Veldhoen, M., and Martin, B., Semin. Immunol., 2007, vol. 17, pp. 353-361. 